\title{
Indoor Positioning Technology Based on Multipath Effect Analysis
} Bing $\mathrm{Xu}^{1, \mathrm{a}}$, Feng Hong ${ }^{2, \mathrm{~b}}$, Xingyuan Chen ${ }^{3, \mathrm{c}}$, Jin Zhang ${ }^{2, \mathrm{~d}}$, Shikai Shen ${ }^{1, \mathrm{e}}$

${ }^{1}$ the College of Information Technology Kunming University, Kunming, YunNan, 650214, China

${ }^{2}$ College of Information Science \& Engineering, Ocean University of china, Qingdao, Shandong, 266001, China

${ }^{3}$ College of Marine Geoscience, Ocean University of china, Qingdao, Shandong, China

axubingqd@163.com, bgray.hong@163.com, '95430601@qq.com, ${ }^{d} 23033894 @ q q . c o m,{ }^{e} 4452158$ 98@qq.com,

Keywords: Multipath Effect; Positioning technology; Perpendicular Intersection(PI)

Abstract. Exsiting indoor localization approaches have poor accuracy because of multipath effect. In this work, we utilize Perpendicular Intersection(PI) means, establishing the model of multipath effect on signal propagation. The results show that multipath effect can be overcomed by PI and high accuracy is achieved.

\section{Introduction}

Sensor network system derives the raw data to determine the high-level application information contained in the application to complete the task by means of collecting various nodes within the network of the original data. Among them, the location information of node (or perceptually relevant raw data) is an important basis for the completion of application tasks sensor network system $[1,2]$. The node positioning technology is the key research of sensor networks.

Currently, GPS positioning technology provides basic wireless positioning means, which can achieve meter level positioning accuracy in the outdoor environment. Detecting Wi-Fi or RFID signal intensity is the most commonly used way of Indoor positioning, and then converted into a distance from the signal intensity information, and then determine the location of the relevant node. However, the positioning accuracy of these two methods can only reach the level of more than meters[3], so that cannot meet the need for a considerable part of the indoor precise positioning applications, such as patient tracking hospital environment, key goods factory premises located inside or smart home for the user providing location-based services.

Indoor positioning accuracy is low because that the indoor radio signal propagation is reflected by walls or other obstructions, resulting that the signal received by node to be located having a severe multipath interference effect, which is also simply called multipath effect. Multipath effect causes the signal received by the node to be located is not an effective response to distance its beacon node, so that the positioning accuracy of the results is poor.

So, how to solve multipath effect for the problem at the node cost constraints, to achieve indoor high-precision positioning has important scientific significance and application value.

This paper designed and completed an indoor environment construction method of rotating beacon nodes, and then researched on the laws of indoor radio signal propagation and multipath signal propagation effects on this basis.

\section{Recent Researches on Positioning Technology}

Orientation of sensor network node is always a hot issue of sensor network technology. At present, the sensor network positioning technology can be divided into three categories: distance-based location technology, distance-independent positioning technology, and positioning technology based on frequency analysis.

Positioning technology based on distance measures the position of the nodes to be located by 
physical distance or azimuth angle measurements between adjacent nodes. Distance-based positioning technology can be classified according to different ranging methods, typically ranging methods are: Based on the Time of Arrival (TOA) [4], the Time Difference of Arriva (TDOA) [5], the Angle of Arrival (AOA) [6] and wireless signal strength (RSS) [3], and so on.

Existing distance-based positioning technology cannot solve the indoor problems of low-cost, high-precision positioning. The methods based on AOA, TOA and TDOA are mainly limited by cost; the question of RSS method is that its positioning accuracy reaches only meter level, which does not meet the requirements of high accuracy indoor location.

Distance-independent positioning technology such as Hop-Count which only uses neighbor nodes for position calculation does not use absolute distance or angle information or any additional hardware. This technology includes centroid algorithm [7], DV-Hop algorithm [8] and APTT algorithm [9] and so on.

Distance-independent positioning technology has no need for measuring the absolute distance or orientation of nodes, reducing the need for node hardware, and positioning performance is less influenced by environmental factors. However, the positioning error of this approach is relatively large, which is not suitable for indoor positioning accuracy.

The location technology based on frequency analysis such as SpinLoc, reaches a low-cost and high accuracy indoor positioning results. It weakens the impact of multipath effects from Doppler frequency offset analysis. However, multipath effects will also affect the results of Doppler frequency analysis. Because SpinLoc experiment is completed in a relatively open center area of indoor parking, multipath effects on Doppler frequency analysis is less affected. If we can carry out effective analysis of indoor multipath effects, indoor positioning accuracy will be further improved.

\section{Perpendicular Intersection(PI) algorithm}

In our experiment, when the mobile beacon moves along a straight line, the largest RSSI value received by a sensor node often, if not always, corresponds to the point on the line that is closest to the node. Theoretically, the point should be the projection of the sensor node on the trajectory; this node can be located as the intersection point of two perpendiculars that cross the mobile beacon's trajectory over the two projections[10].

In order to illustrate how PI works, we show an example in Fig.1, where a mobile beacon traverses the region while broadcasting beacon packets periodically. A beacon packet contains the coordinates of the position of the mobile beacon. The solid black lines in Fig. 1 from the trajectory of the mobile beacon, with the arrows denoting its moving directions .The mobile beacon (in red) starts At point $P_{1}$, changes its direction at point $P_{2}$, and stops at point $\mathrm{P}_{3}$. By combining the trajectory with the virtual line $P_{1} P_{3}$, we obtain a virtual triangle $\Delta P_{1} P_{2} P_{3}$ (we call it VT from now on).

Let $R$ be the transmission range of mobile beacon. To ensure that all the modes in a VT can receive the signals from the beacon, the sides $P_{1} P_{2}$ and $P_{2} P_{3}$ should not be longer than $R$. Meanwhile, the angel $\theta$ between the two lines should satisfy $0<\theta \leq 3 / \pi$.

Suppose the five nodes (in blue) in FIg.1 are located using PI. We use node $N(x, y)$ as an example. The mobile beacon starts at point $P_{1}$ and broadcasts the start signal with its current location. Node $N$ records the start position when it Hears the start signal. Along its trajectory from $P_{1}$ to $P_{2}$, the mobile beacon broadcasts beacon packets periodically with its current location. Node $N$ receives all the beacon packets, and records the one with the largest RSSI value. When the mobile beacon arrives at $P_{2}$, it broadcasts a stop signal with its current location. When node $N$ receives the stop packet, it knows that the mobile beacon has just finished traversing the line from $P_{1}$ to $P_{2}$.

The recorded position is the position where the beacon packet with largest RSSI value broadcast. We label the recorded position as $A\left(x^{\prime}, y^{\prime}\right)$.

Line segment $N A$ is the shortest one among all the line segments connecting node $N$ and any point on line $P_{1} P_{2}$. In other words, point $A$ is the projection of node $N$ on line $P_{1} P_{2}$. And we have: 


$$
\frac{y_{2}-y_{1}}{x_{2}-x_{1}} \times \frac{y-y^{\prime}}{x-x^{\prime}}=-1
$$

Similarly, when the mobile beacon moves from $P_{2}$ to $P_{3}$, another position

$B\left(x{ }^{\prime}, y\right.$ ” $)$ is recorded which is the projection of node $N$ on line $P_{2} P_{3}$. Thus we have:

$$
\frac{y_{3}-y_{2}}{x_{3}-x_{2}} \times \frac{y-y^{\prime \prime}}{x-x^{\prime \prime}}=-1
$$

By solving Formulas (1) and (2), we can compute the coordinate $(x, y)$ of node $N$ :

$$
\left(\begin{array}{l}
x \\
y
\end{array}\right)=\left(\begin{array}{cc}
x_{2}-x_{1} & y_{2}-y_{1} \\
x_{3}-x_{2} & y_{3}-y_{2}
\end{array}\right)^{-1} \times M
$$

Where

$$
M=\left(\begin{array}{cccc}
x_{2}-x_{1} & y_{2}-y_{1} & 0 & 0 \\
0 & 0 & x_{3}-x_{2} & y_{3}-y_{2}
\end{array}\right)\left(\begin{array}{l}
x^{\prime} \\
y^{\prime} \\
x^{\prime \prime} \\
y^{\prime \prime}
\end{array}\right)
$$

In the above process, we only contrast the RSSI values and utilize the geometric relationship among the nodes and the beacon to do localization. The location calculation does not require any absolute values of RSSI, nor does it base on any signal propagation model. In this way, PI is expected to avoid the potential errors generated from the translations from RSSI to distances.

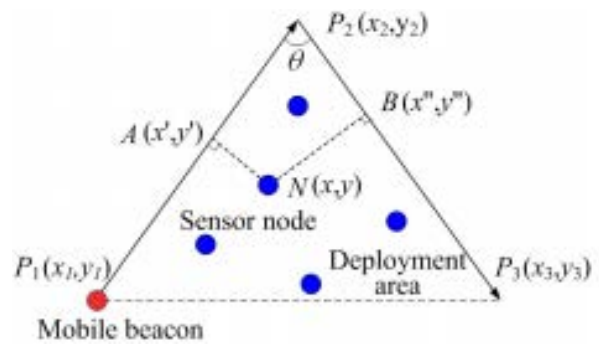

Fig.1 An example of PI scheme

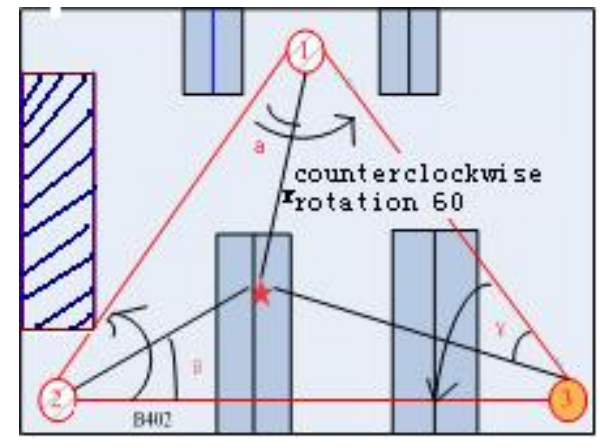

Fig.2 Experimental deployment of indoor multipaph analysis and positioning effect relationship analysis

\section{Indoor positioning technology model based on multipath effects analysis}

In the experiment, 3-4 rotating beacons are deployed in indoor environments(78m2, with tabales) and put dozens of experiments into practice in different locations. The diagram of three rotating beacons is shown in the Fig.2. The (1)(2)(3) are the locations of rotating beacon, the red five-pointed star within the triangle is the location of node to be located.

Three beacons periodically emit beacon packets containing the current angle during the rotation. The frequency is $30 \mathrm{~Hz}$. The signal strength of beacon packet from the three beacons which is recorded by the node to be located is regarded as the basis for multipath analysis and positioning analysis. The strength curves of three beacon nodes' beacon packets in one experiment of node record to be located, which were processed by low-pass filter, are shown in picture two.

The signal strength from the three beacons recorded by the node to be located in one experiment is shown in Fig.3. The signals in the strength curves have been processed by low-pass filter.

The maximum angle of signal strength received by nodes to be located within a window can be calculated according to Fig.3, for example, the angle marked in red in Fig.3. Because the strength of received signal is larger, the distance between receiving point and firing point is shorter, the maximum angle may be the sight angle from receiving point to firing point. The angle's 
propagating line of beacon packet from the sending node and the propagating line reflected by walls are shown in Fig.4.

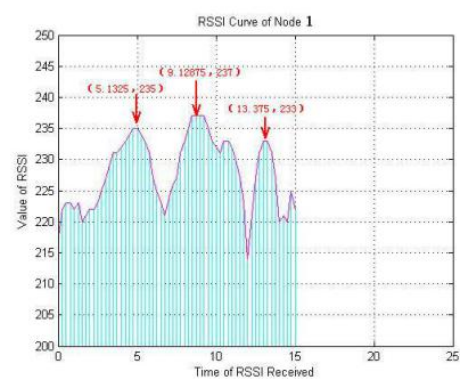

(a)

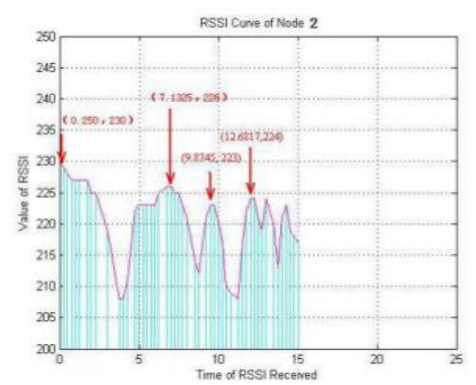

(b)

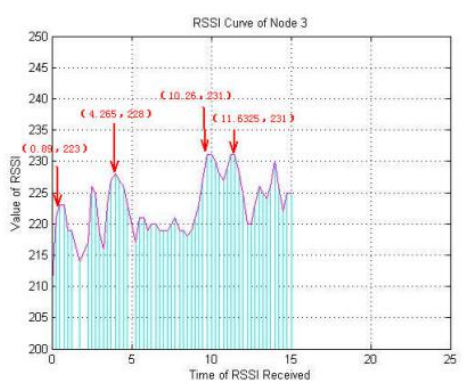

(c)

Fig.3 Signal intensity curve of beacon packets received by nodes to be located after low-pass filtering. (a) beacon node 1 (b) beacon node 2 (c) beacon node 3
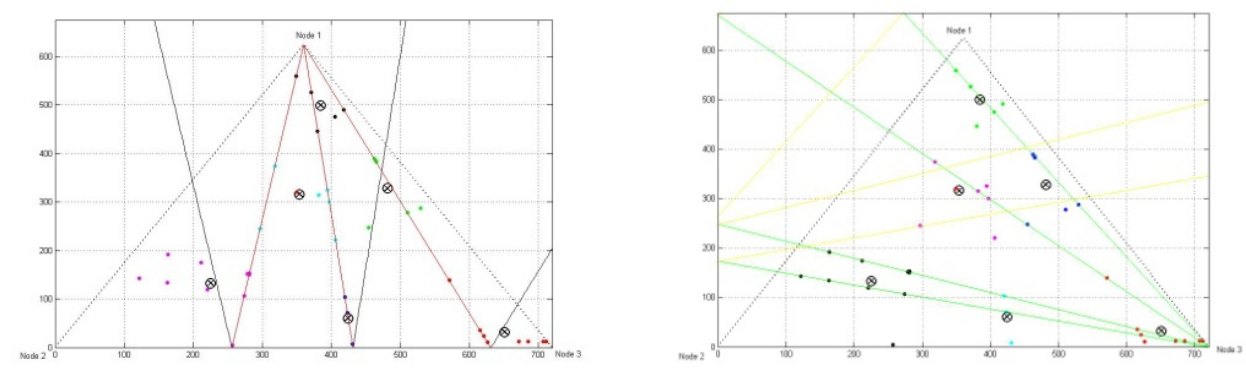

Fig. 4 The beacon packet's maximum angle propagation lines and reflection lines. (a) From beacon node 1. (b) From beacon node 2.

\section{Conclusion}

In this paper, we established the impact model of multipath effects on signal propagation with PI method according to the characteristic points and indoors wall reflection. In order to examine this method, we perform a laboratory experiment. We took a low-pass filter for the preprocessing of beacon packet signals, used the maximum angle propagation lines chart for the positioning analysis, and finally determine the position of firing point. The positioning error is $2 \mathrm{~m}$ or less, which demonstrates that PI method can overcome the multipath effect to execute high-precision indoor positioning.

\section{Acknowledgement}

This work was financially supported by the Kunming University Foundation (XJL14005), Innovation Program of Internet of things application technology of Kunming University (2015CXTD04). All the persons involved in the research projects are thanked for their help. 


\section{References}

[1] M. Maroti, B. Kusy, G Balogh, P. Volgyesi, A Nadas, K. Molnar, S. Dora, and A. Ledeczi, Radio interferometric geolocation, in Proc. of 3rd ACM International Conference on Embedded Networked Sensor Systems(SenSys), November 2005.

[2] B.Kusy, A.Ledeczi, and X.Koutsoukos, Tracking mobile nodes using RF Doopler shifts, in Proc. of 5th ACM International Conference on Embedded Networked Sensor Systems(SenSys), November 2007.

[3] P. Bahl and V.N.Padmanabhan, RADNR:An In-Building RF-Based User Location and Tracking System, Proceedings of IEEE INFOCOM, 2002.

[4] A. Savvides, C. Han, and M.B. Srinvastava. Dynamic finegrained localization in ad-hoc networks of sensors. Proceedings of ACM MobiCom, 2001.

[5] A. Savvides, H. Park, and M.Srivastava. The bits and flops of the n-hop multilateration primitive for node localization problems. Proceedings of ACM WSNA, 2002.

[6] D. Niculescu and B. Nath. Ad hoc positioning system (aps) using aoa. Proceedings of IEEE INFOCOM, 2003

[7] Nasipuri A. Das S R. On-Demand Multipath Routing far Mobile Ad Network, In:Proc. Intl Confon Computer Commuttication and Nerworks(ICCCN), 64-70, Oct.1999.

[8] Kaplan L M, Molnar P, Le Q, Bearings-only target localization for an acoustical unattended ground sensor network[C]. Orlmdo, FL, United States, 40-51,2001.

[9] D. Niculescu and B.Nath. Dv based positioning in ad hoc networks. Journal of telecommunication Systems, 22(1):267-280, January 2003.

[10] Zhongwen Guo, Ying Guo, Feng Hong, Xiaohui Yang, Yuan He, Feng Yuan, Yunhao Liu, Perpendicular Intersection: Locating Wireless Sensors with Mobile Beacon, In Proceedings of the 29th IEEE Real-Time System Symposium(RTSS 2008). Dec 2008. 